\title{
Review
}

\section{The "book" medium and scientific editorial communication: prospects and ongoing changes}

\author{
Laura Massoli
}

\section{G. Vitiello, Il libro contemporaneo, Editrice Bibliografica, Milano (2009)}

\begin{abstract}
The volume "Il libro contemporaneo" (The Contemporary Book) by Giuseppe Vitiello offers a global view of the "book" as a model and as an instrument of communication and for learning in the society of knowledge; it specifically deals with scientific editorial communication, through a complete and systematic reconstruction of the bodies involved, of the production and dissemination processes, also in the framework of the technological changes pushed by new media. In particular, the author critically analyzes some relevant aspects such as the role played by the journal as the most relevant mean for scientific knowledge dissemination, the scientific writer figure, the strengthening of large publishing groups and the challenge open access implies.
\end{abstract}

The volume Il libro contemporaneo (The Contemporary Book) by Giuseppe Vitello (Editrice Bibliografica, 2009) has a leading thread and presents a unitary view - a view of a book as a complex communication and learning cultural model which survives and permeates the different forms, digital and/or paper ones, taken by this kind of "technology" and which characterizes the entire production and dissemination process.

The choice of this unitary view, the initial and decisive definition of a book as a "technology of freedom" allows the author to tie and to insert aspects and paths into a system which apparently are very different: the historical reconstruction and critical analysis, in the first part, of the "book" medium in the various communication theories and in the complex process of the cultural industry, now turning towards converging and mediamorfosis models; the description of circuits of production and dissemination, whether commercial or not, which are dealt with in the second part; the analysis of the scenario of European book national policies, which concludes the third and last part.

The books gives special attention to the field of scientific editorial communication - a sector which has hardly been the subject of a systematic and complete analysis - as it happens in this book. With no doubts, one can say that scientific editorial communication is analyzed here for all of its components: production, procedures, roles and figures involved. And this completeness and systematization, which does not rule out the specific analysis of some of the most innovative aspects, such as the "scientific writer", is one of the most interesting and significant parts of this book.

In particular, in a specific chapter, the author dwells upon the role played by the scientific journal as an instrument to spread research findings in a sector marked by different and often contrasting tones: the large publishing groups and the advent of 'open access'. The book reports, also from a historical viewpoint, the rising of scientific publishing up to the present-century concentration and the intuition - a very premonitory one - regarding on-line publishing: in the scientific field, thanks to digitalization, "nothing has been like it was before".

Particularly interesting are the remarks on the "excessive power" of scientific editors and on how their role has changed in the internet era: the analysis considers both horizontal integrations - typically acquisitions - and vertical integrations, i.e. between the production link and the content distribution link. As we moved from printed material to electronic supports, scientific editors acquired a predominant position, as Vitiello underlines, centralizing the negotiation directly with libraries and avoiding both the apportionment of the offer and the intermediation of subscription agencies. This process, defined disintermediation, is interpreted not as a change on its own, but as related to the aggregation phenomena that, on the other hand, involve libraries themselves. To face the growing pressure from editorial groups 
which are more and more united, libraries form consortia, giving birth to a phenomenon, as it is defined in the book, of "bilateral oligopoly" which sees the contraposition of two power centres: on the one hand library consortia, and on the other hand large scientific publishing companies.

In a systemic perspective, the book comprises an interesting and complementary in-depth analysis of the phenomenon of alternative publishing and of open archives, where open access does not simply designates "a professional platform, but also a political and social mobilization which crosses the topical contemporary issues of a sustainable development founded on a knowledge society" (p. 308).

The author reconstructs the two circulation patterns for open access scientific publications: the first features a direct mechanism, and an author-reader unidirectional relation, without evaluation and control by an editorial office; the second revalues the role by the editor as an intermediary and an aggregating agent. The proposed analysis follows an interesting economic approach which evaluates the spill-over costs for both libraries and institutions which are references to researchers.

As mentioned, Vitiello proposes an innovative reflection on the role played by scientific writers who in general are hardly considered, as we always are mainly focussed on literary writers. After a brief review on the "biodiversity of researchers" (p. 124), marked by mechanisms such as peer reviewing and impact factor (which are both also reconstructed in the book), the analysis turns to the aspect, which is much more topical and stimulating, to my mind, of the role played by the author and of the related elements of authorship and certification of the work, in the digital era of Wikipedia and of collective intelligence. Indeed, in the communication networks, as the author correctly underlines, it becomes much less obvious to define individual ownership, which may become a collective exercise, where certification can be exerted not only by editors but also by users' consensus.

The influence of communication networks is also used as an interpretation key in the chapter devoted to the free use for scientific purposes: the analysis carried out highlights both the regulating-sanctioning framework which has recently inserted the subject into a system, and the comparative approach with the models followed in other European countries and in the US, and finally also the difficulties, but also the opportunities that new media guarantee, between the danger of copyright infringements and the potential dissemination of culture and science.

To conclude with, a relevant chapter is the one devoted to the role of libraries, even in the perspective of the guarantee of the access to information and scientific literature and the protection of public interest. In this regard, Vitiello says - and one cannot but agree with him - that plurality, which public libraries should promote, "is not the expression of an apportionment of knowledge, but it is a positive selection, aiming at promoting, within the library collection itself, a dialogic relation between ideas and at expressing the contents through a stimulating and critical public narration" (p. 207).

Translated by Massimo Caregnato

\section{Author}

Laura Massoli, who holds a PhD in Sciences of Communication and Public Relations, is head of Unit at the Public Administration Department - Presidency of the Council of Ministers. Having obtained her degree in Sciences of Communication from the University of Siena, she attended a master's degree in Public Communication and Management of multimediality at the University of Rome La Sapienza from which she subsequently obtained her PhD. She has worked for the Communications Regulatory Authority and ENEA (Agency for New Technologies, Energy and Environment) and has collaborated with various Italian (University La Sapienza, CNR - National Council for Research, Ministry of Economy) and international (European Schoolnet, IBM) institutions, participating to projects in the field of public communication and innovation. She is the author (together with F. Faccioli and L. D'Ambrosi) of "Voci della Ribalta: Comunicazione sociale, processi inclusivi e partecipazione", ESI 2007 and of various articles on the issues of communication and new technologies. E-mail: laura.massoli@libero.it.

How TO CITE: $\quad$ L. Massoli, The "book" medium and scientific editorial communication: prospects and ongoing changes, Jcom 08(03) (2009) R01. 